\title{
Uso de la técnica de relajación térmica para la medición de calor específico de láminas recubiertas con $\mathrm{TiO}_{2}$
}

\author{
Use of thermal relaxation technique for measuring of specific heat of sheets coated \\ with $\mathrm{TiO}_{2}$
}

\author{
F. Gordillo-Delgado iD, D. F. Valencia-Grisales iD, J. A. García-Giraldo iD
}

\begin{abstract}
Specific heat $\left(C_{p}\right)$ is a necessary thermal parameter for describing heat transport phenomena, related to adaptation to temperature changes. For this reason, it is important in the construction, glue, thermal insulator and electronic device industry. In this work, a thermal relaxation system was used to determine the $\mathrm{C}_{\mathrm{p}}$ of $\mathrm{Ti}$ sheets, coated with $\mathrm{S}_{-} \mathrm{TiO}_{2}$ by Plasma Electrolytic Oxidation (EPO). Each sample was placed inside a vacuum chamber that reaches around $10^{-2}$ Torr and was heated by the incidence of a laser beam of $450 \mathrm{~nm}$ wavelength; an infrared (IR) thermometer was located at the bottom of the sample and the data obtained was sent to a computer using a programmable development card. The validation of the system was done by comparing the results with those reported by other authors for zinc, tungsten, titanium and steel. The $C_{p}$ of the OEP coated Ti samples, which were obtained using an electrolyte with two concentrations of thiourea $\left(\mathrm{CH}_{4} \mathrm{~N}_{2} \mathrm{~S}\right)$ and with a voltage applied between the electrodes at $2 \mathrm{kHz}$ with useful cycles of 10 $\%, 20 \%$, and $30 \%$, were measured and compared in order to study the influence of growth parameters on the thermal characteristics of the material. The results of the analysis of variance of the data led to find significant differences, with a confidence level of $95 \%$, related to the variation in the concentration of $\mathrm{CH}_{4} \mathrm{~N}_{2} \mathrm{~S}$. This is an indication of the potential use of thermal relaxation technique to determine particularities of this type of coatings.
\end{abstract}

Index Terms - Specific heat; infrared thermometry; thermal relaxation; coatings by $\mathrm{PEO}$

Este manuscrito fue enviado el 7 de noviembre de 2018 y aceptado el 23 de septiembre de 2019.

Este proyecto fue financiado por la Universidad del -Quindío, a través del proyecto 924 "Evaluación de características mecánicas y térmicas de elementos estructurales elaborados con guadua Angustifolia Kunth y con un adhesivo sintetizado con aceite de higuerilla", ejecutado por el Grupo de Investigación en Ciencia Aplicada para el Desarrollo de la EcorregiónGICADE, adscrito al IIC.

F. Gordillo-Delgado, líder del GICADE, docente del Programa de Tecnología en Instrumentación Electrónica de la Universidad del Quindío, $\mathrm{Ph} . \mathrm{D}$ del Centro de Investigación en Ciencia Aplicada y Tecnología avanzada - CICATA del IPN, México (fgordillo@uniquindio.edu.co).

D. F. Valencia-Grisales, integrante del GICADE, ingeniero electrónico de la Universidad del Quindío (dfvalenciag@ uqvirtual.edu.co).

J. A. García-Giraldo, integrante del GICADE, ingeniero electrónico de la Universidad del Quindío (jagarciag_1@ uqvirtual.edu.co).
Resumen- El calor específico $\left(C_{p}\right)$ es un parámetro térmico necesario para la descripción de fenómenos de transporte de calor, relacionado con la adaptación a cambios de temperatura. Por esta razón es importante en la industria de la construcción, de pegantes, de aislantes térmicos y de dispositivos electrónicos. En este trabajo, se utilizó un sistema de relajación térmica para determinar el $C_{p}$ de láminas de $\mathrm{Ti}$, recubiertas con $\mathrm{S}_{-} \mathrm{TiO}_{2}$ por Oxidación Electrolítica con Plasma (OEP). Cada muestra se ubicó dentro de una cámara de vacío que alcanza alrededor de $10^{-2}$ Torr y fue calentada por la incidencia de un haz de luz láser de $450 \mathrm{~nm}$ de longitud de onda; se ubicó un termómetro infrarrojo (IR) en la parte inferior de la muestra y los datos obtenidos se enviaron a un computador, usando una tarjeta de desarrollo programable. La validación del sistema se hizo comparando los resultados con los reportados por otros autores para zinc, wolframio, titanio y acero. El $C_{p}$ de las muestras de Ti recubiertas por OEP, que fueron obtenidas usando un electrolito con dos concentraciones de tiourea $\left(\mathrm{CH}_{4} \mathrm{~N}_{2} \mathrm{~S}\right)$ y con tensión aplicada entre los electrodos a $2 \mathrm{kHz}$ con ciclos útiles de $10 \%, 20$ $\%$, y $30 \%$, se midió y se comparó con el fin de estudiar la influencia de los parámetros de crecimiento en las características térmicas del material. Los resultados del análisis de varianza de los datos llevaron a encontrar diferencias significativas, con un nivel de confianza del $95 \%$, relacionadas con la variación en la concentración de $\mathrm{CH}_{4} \mathrm{~N}_{2} \mathrm{~S}$. Esto es un indicio del potencial uso de la técnica de relajación térmica para determinar particularidades de este tipo de recubrimientos.

Palabras claves - Calor específico; termometría infrarroja; relajación térmica; recubrimientos por OEP

\section{INTRODUCCIÓN}

L as características térmicas de los sólidos son importantes en el ámbito industrial y en las ciencias de los materiales. En particular, la información sobre el calor específico, $C_{p}$, que expresa la cantidad de calor necesario para que la temperatura de una muestra de volumen constante aumente en un grado [1], sirve para describir los fenómenos de transporte de calor en un material.

Los equipos comerciales para la medición de parámetros termofísicos son costosos, por lo que es ventajoso el desarrollo de sistemas más simples [2, 6-8]; aunque se han propuesto varios métodos y cálculos analíticos que permiten la 
determinación de los parámetros termofísicos, en diferentes condiciones iniciales y rangos de temperatura, la técnica de relajación térmica para la obtención del $C_{p}$ de muestras sólidas, de tamaño pequeño, es una alternativa sencilla y bien documentada [3, 4]. Este método se basa en perturbar, mediante iluminación constante, el estado de equilibrio termodinámico de la muestra suspendida adiabáticamente dentro de una celda al vacío y en medir la variación de su temperatura absoluta $T(t)$ en función del tiempo [5]. El calor es generado por un haz de luz láser que impacta la superficie frontal de la muestra, bajo condiciones específicas de presión; el comportamiento de la diferencia de temperatura entre esta cara y la opuesta permite obtener el $C_{p}$ de la muestra, a través de la solución analítica de la ecuación de difusión del calor [2].

La instalación experimental del método de relajación térmica es de bajo costo y el formalismo físico-matemático que se emplea para analizar el comportamiento de la temperatura cuando la muestra sufre calentamiento y enfriamiento es relativamente simple [1,9]. Esta técnica se ha utilizado en los últimos años para la caracterización de diferentes materiales semiconductores, alimentos, maderas, zeolitas, arcillas, y polímeros, entre otros [1].

El $C_{p}$ de un material es característico de cada sustancia y depende de su estructura interna y composición. En este trabajo, se reporta la determinación del calor específico de sólidos mediante un sistema implementado; en particular, se obtuvo reproducibilidad y se compararon los valores obtenidos con los reportados para materiales bien conocidos. Se describe la instrumentación electrónica que se utilizó para optimizar la exactitud y precisión de las mediciones; para esto se garantizó que la temperatura censada fuera adquirida y guardada como un dato en un tiempo determinado, a través de la programación en las interfaces de la tarjeta de desarrollo.

\section{MetodologíA}

En la Fig. 1 se presenta el esquema general del montaje experimental. La superficie frontal de la muestra se radia con un haz de luz láser de longitud de onda de $450 \mathrm{~nm}$ y se mide el gradiente de temperatura utilizando termometría infrarroja. La muestra se coloca dentro de una cámara cilíndrica de acero inoxidable de $0,4 \mathrm{~m}$ de alto y $0,25 \mathrm{~m}$ de diámetro, conectada a una bomba de vacío Leybold Trivac D 2,5 E, con la que se alcanza una presión de alrededor de $10^{-2}$ Torr dentro de la cámara.

La temperatura se midió con un termómetro infrarrojo de referencia TM-908 LUTRON, ubicado en la parte trasera de la muestra. Los datos se registraron a través de una tarjeta de desarrollo programable, a una velocidad sincronizada entre la recepción de la temperatura y el envío de datos hacia el computador. Esto evitó pérdidas o latencia de información, que provocarían una toma de datos errónea.

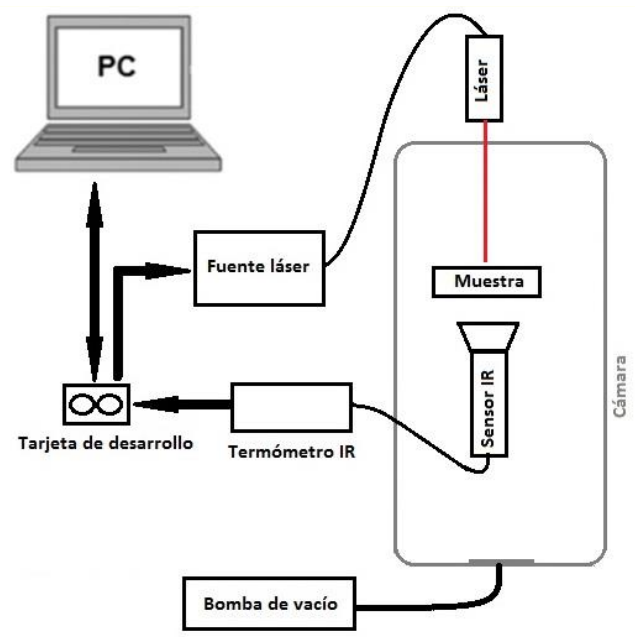

Fig. 1. Esquema del montaje experimental.

El área de la superficie frontal de la muestra (por donde incide el haz de luz láser) se tiñó con una fina capa de pintura de carbón para acercarse a condiciones de calentamiento uniforme y transferencia de calor unidireccional. La luz absorbida por el sólido se convierte, en parte o en su totalidad, en calor mediante procesos no radiativos de desexcitación [10].

Si $Q$ es el calor generado por la radiación sobre la muestra, de densidad $\rho$ y volumen $V$, que da lugar a una variación de su temperatura $\Delta T\left(T_{\text {final }}-T_{\text {inicial }}\right)$, la capacidad calorífica por unidad de volumen, $C$, está dada por (1):

$$
Q=C V \Delta T=m C_{p} \Delta T=\rho C_{p} V \Delta T
$$

Esta variación depende del tiempo de exposición a la fuente de calor. En la técnica de relajación térmica, teniendo en cuenta que la muestra se suspende en el interior de una cámara con un hilo de baja conductividad térmica, las pérdidas de calor por conducción se aproximan a cero. Por otro lado, la transferencia de calor por radiación se describe a través de (2):

$$
R=A \varepsilon \sigma\left(T^{4}-T_{o}^{4}\right)
$$

Donde $A$ es el área de la sección transversal de la muestra, $\varepsilon$ es el coeficiente de emisividad (se supone $\varepsilon \approx 1$ ) y $\sigma$ es la constante de Stefan-Boltzmann. Si las variaciones de temperatura $\Delta T$ son cercanas a la temperatura ambiente, $T_{0}$, se tiene que:

$$
R=4 A \epsilon \sigma\left(T_{0}^{3} \Delta T\right)
$$

Por otro lado, de acuerdo con la ley de enfriamiento de Newton para la convección, la pérdida de calor de un cuerpo es proporcional a la diferencia de temperatura entre el cuerpo y sus alrededores, de tal forma que (4) describe este fenómeno.

$$
\frac{d Q}{d t}=h A\left(T_{\text {muestra }}-T_{\text {fluido }}\right)
$$

Donde $h$ es el coeficiente de convección o de transmisión superficial. 
Derivando (1) y hallando su solución, con condiciones iniciales $\Delta T(0)=0$, se tiene (5):

$$
\Delta T(t)=\frac{P_{o}}{\beta}\left[1-\exp ^{(-t / \tau)}\right]
$$

Donde $\beta$ es un parámetro relacionado con la temperatura inicial, descrito en (6).

$$
\beta=A\left(4 \epsilon \sigma T_{0}^{3}+h\right)
$$

El tiempo de relajación térmica, $\tau$, está dado por (7):

$$
\tau=\frac{L C}{2\left(4 \epsilon \sigma T_{o}^{3}+h\right)}
$$

El parámetro $h$ no se relaciona con las propiedades de la muestra, únicamente depende de la geometría del dispositivo de medición y de las características del fluido en el que se sumerge la muestra, que en este caso es aire.

Cuando la luz no irradia la muestra, en (5) cambia, porque la temperatura de la muestra se satura en un valor $T_{0}=P_{0} / \beta \mathrm{y}$ luego disminuye de acuerdo con (8).

$$
\Delta T(t)=\frac{P_{o}}{\beta}\left[\exp ^{(-t / \tau)}\right]
$$

Cuando la cantidad de aire es muy pequeña en el interior de la cámara, en la que se coloca la muestra, la transferencia de calor por convección tiende a cero y por lo tanto el tiempo de relajación térmica sólo es afectado por la radiación. Ya que en este caso el parámetro $h$ se desprecia, a partir de (7) la capacidad calorífica se puede expresar como (9):

$$
C=\frac{8 \epsilon \sigma T_{o}^{3} \tau_{R}}{L}
$$

Esta técnica fue usada en este trabajo para medir el $C_{p}$ de láminas recubiertas con $\mathrm{TiO}_{2}$, que se obtuvieron por oxidación electrolítica con plasma (OEP) de sustratos de titanio (Ti) de dimensiones $10 \mathrm{~mm}$ x $10 \mathrm{~mm}$ x $1 \mathrm{~mm}$, utilizando una tensión eléctrica de $340 \mathrm{~V}$ dc, con el fin de determinar la influencia del electrolito usado en este método electroquímico, que puede llevar al depósito de impurezas donadoras de electrones en la capa de óxido que se forma y a la disminución del ancho de banda de energía prohibida del material.

\section{TABLA I}

CARACTERÍSTICAS DE LAS MUESTRAS DE $\mathrm{TIO}_{2}$ CON INCORPORACIÓN DE S.

\begin{tabular}{cc}
\hline \hline Ciclo útil (\%) & Electrolito \\
\hline 10 & $0,1 \mathrm{M} \mathrm{H}_{2} \mathrm{SO}_{4} / 52 \mathrm{mM} \mathrm{CH}_{4} \mathrm{~N}_{2} \mathrm{~S}$ \\
& $0,1 \mathrm{M} \mathrm{H}_{2} \mathrm{SO}_{4} / 79 \mathrm{mM} \mathrm{CH}_{4} \mathrm{~N}_{2} \mathrm{~S}$ \\
20 & $0,1 \mathrm{M} \mathrm{H}_{2} \mathrm{SO}_{4} / 52 \mathrm{mM} \mathrm{CH}_{4} \mathrm{~N}_{2} \mathrm{~S}$ \\
& $0,1 \mathrm{M} \mathrm{H}_{2} \mathrm{SO}_{4} / 79 \mathrm{mM} \mathrm{CH}_{4} \mathrm{~N}_{2} \mathrm{~S}$ \\
30 & $0,1 \mathrm{M} \mathrm{H}_{2} \mathrm{SO}_{4} / 52 \mathrm{mM} \mathrm{CH}_{4} \mathrm{~N}_{2} \mathrm{~S}$ \\
& $0,1 \mathrm{M} \mathrm{H}_{2} \mathrm{SO}_{4} / 79 \mathrm{mM} \mathrm{CH}_{4} \mathrm{~N}_{2} \mathrm{~S}$ \\
\hline
\end{tabular}

En este trabajo, se utilizaron las 6 muestras descritas en la Tabla I, crecidas con OEP a frecuencia de $2 \mathrm{kHz}$, con diferente ciclo útil, usando un electrolito formado por $0,1 \mathrm{M}$ de $\mathrm{H}_{2} \mathrm{SO}_{4} \mathrm{y}$ dos concentraciones de $\mathrm{CH}_{4} \mathrm{~N}_{2} \mathrm{~S}$.

\section{RESULTADOS Y DISCUSIÓN}

A. Medición del calor específico de muestras de zinc (Zn), wolframio $(W)$, titanio $(T i)$ y acero.

Partiendo de (5) y (8), que describen respectivamente la evolución de la temperatura cuando la muestra es irradiada con el haz de luz láser y cuando se enfría hasta regresar a su temperatura inicial, se obtiene el tiempo de relajación térmica. A través de (10), considerando únicamente transferencia de calor por radiación y teniendo en cuenta la masa y el volumen de las muestras que aparecen en la Tabla II, se halla la capacidad calorífica y el calor específico.

En la Fig. 2, se muestran las curvas de evolución de temperatura, en ambiente de vacío, de muestras de $\mathrm{Zn}, \mathrm{W}$, Ti y acero, durante los procesos de calentamiento y enfriamiento, respectivamente. El tiempo de relajación y el calor específico de las muestras son presentados en la Tabla III.

TABLA II

DiMENSIONES DE LAS MUESTRAS DE ZN, W, TI, Y ACERO.

\begin{tabular}{ccccc}
\hline \hline $\begin{array}{c}\text { Muestra } \\
(99 \%)\end{array}$ & $\begin{array}{c}\text { Largo } \\
(\mathrm{mm}) \\
\pm 0,01 \\
\mathrm{~mm}\end{array}$ & $\begin{array}{c}\text { Ancho } \\
(\mathrm{mm}) \\
\pm 0,01 \\
\mathrm{~mm}\end{array}$ & $\begin{array}{c}\text { Espesor } \\
(\mathrm{mm}) \\
\pm 0,01 \\
\mathrm{~mm}\end{array}$ & $\begin{array}{c}\text { Masa } \\
(\mathrm{g}) \\
\pm 0,0001 \\
\mathrm{~g}\end{array}$ \\
\hline $\mathrm{Zn}$ & 14,16 & 14,15 & 0,71 & 0,9440 \\
$\mathrm{~W}$ & 15,00 & 14,56 & 0,96 & 3,7050 \\
$\mathrm{Ti}$ & 20,08 & 20,52 & 1,03 & 1,8690 \\
Acero & 19,72 & 18,70 & 0,81 & 2,3400 \\
\hline \hline
\end{tabular}

a)

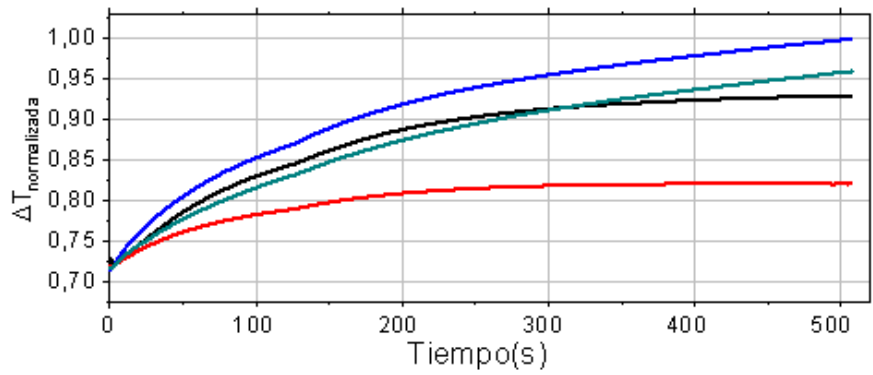

b)

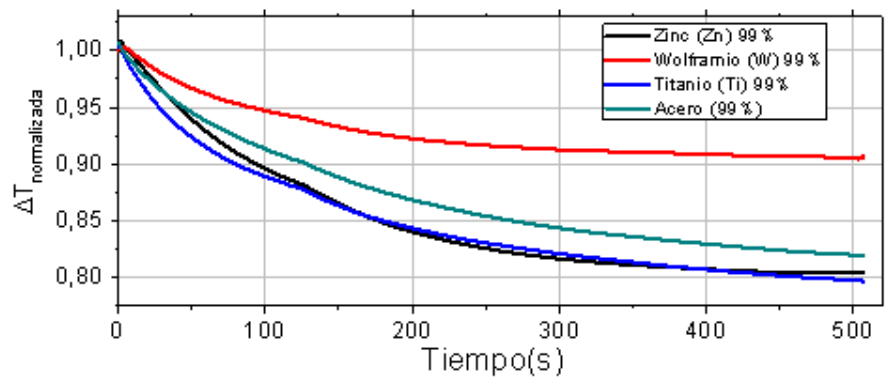

Fig. 2. Gradiente de temperatura de la muestra, en función del tiempo de medición, correspondiente a muestras de $\mathrm{Zn}, \mathrm{W}$, Ti y acero con una pureza del $99 \%$, medida en vacío con a) incidencia de luz láser y b) sin ella. 
TABLA III

VALORES OBTENIDOS Y REPORTADOS DE $C_{P}$ PARA ZN, W, TI, Y ACERO.

\begin{tabular}{ccc}
\hline \hline $\begin{array}{c}\text { Muestra } \\
(99 \%)\end{array}$ & $\begin{array}{c}C_{p} \text { obtenido } \\
\left(\mathrm{J} \cdot \mathrm{g}^{-1} \cdot \mathrm{K}^{-1}\right)\end{array}$ & $\begin{array}{c}C_{p} \text { reportado } \\
\left(\mathrm{J} \cdot \mathrm{g}^{-1} \cdot \mathrm{K}^{-1}\right)\end{array}$ \\
\hline $\mathrm{Zn}$ & $0,38 \pm 0,01$ & $0,389[11]$ \\
$\mathrm{W}$ & $0,11 \pm 0,02$ & $0,133[12]$ \\
$\mathrm{Ti}$ & $0,56 \pm 0,01$ & $0,543[13]$ \\
Acero & $0,46 \pm 0,01$ & $0,460[14]$ \\
\hline \hline
\end{tabular}

De acuerdo con los resultados presentados en la Tabla III, los valores de $C_{p}$ obtenidos con el sistema de relajación térmica son similares a los reportados por otros autores.

B. Difracción de rayos $\mathrm{X}$ de recubrimientos de $\mathrm{TiO}_{2}$ con incorporación de $S$.

En la Fig. 3, se muestran los difractogramas de rayos $\mathrm{X}$ de recubrimientos de $\mathrm{TiO}_{2}$ con incorporación de $\mathrm{S}\left(\mathrm{S}-\mathrm{TiO}_{2}\right)$ que fueron descritos en la Tabla I.
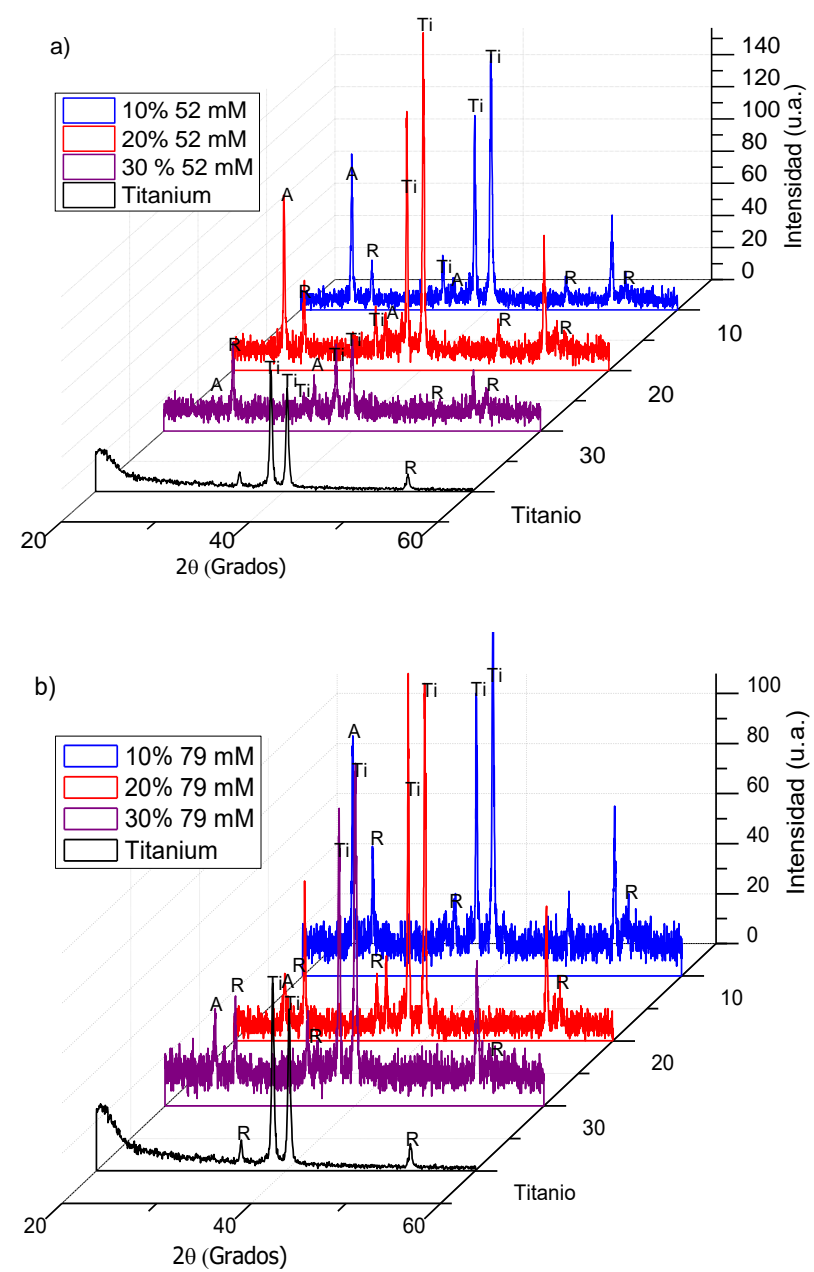

Fig. 3. Difractogramas de rayos $\mathrm{X}$ de las muestras obtenidas a $2 \mathrm{kHz}$, ciclo útil de 10,20 y $30 \%$, con una concentración de electrolito de a) $52 \mathrm{mM}$ y b) de 79 $\mathrm{mM}$ de $\mathrm{CH}_{4} \mathrm{~N}_{2} \mathrm{~S}$.
Se encontraron mezcladas las dos fases del $\mathrm{TiO}_{2}$, Anatasa y Rutilo. En estos difractogramas aparecen tres picos característicos de Anatasa en $25,3^{\circ}, 37,3^{\circ}$, y 47, $6^{\circ}$, asociados a los planos (1 $\left.\begin{array}{lll}1 & 0 & 1\end{array}\right),\left(\begin{array}{lll}0 & 0 & 4\end{array}\right), \mathrm{y}\left(\begin{array}{lll}2 & 0 & 0\end{array}\right)$, respectivamente, y dos picos a la fase Rutilo en $27,5^{\circ}$ y $54,6^{\circ}$, correspondientes a los planos (1 110$)$ y (2 111$)$, respectivamente [15].

A partir de estas curvas se calcularon las fracciones de peso de estas fases cristalinas, utilizando las ecuaciones de Spurr y Myers [16]. Estos valores se muestran en la Tabla IV; se puede observar, que tanto el ciclo útil como el contenido de azufre en el electrolito tienen influencia sobre la estructura nanocristalina de los recubrimientos [17].

TABLA IV

PORCENTAJES DE ANATASA Y RUTILO EN LAS MUESTRAS CRECIDAS POR OEP CON PULSOS DE TENSIÓN A 2 KHZ Y CICLOS DE TRABAJO DE 10, 20 Y $30 \%$, A CONCENTRACIONES DE $\mathrm{CH}_{4} \mathrm{~N}_{2} \mathrm{~S}$ DE $52 \mathrm{MM}$ Y $79 \mathrm{MM}$.

\begin{tabular}{cccc}
\hline \hline $\begin{array}{c}\text { Ciclo útil } \\
(\%)\end{array}$ & $\begin{array}{c}\mathrm{CH}_{4} \mathrm{~N}_{2} \mathrm{~S} \\
(\mathrm{mM})\end{array}$ & $\begin{array}{c}\text { Porcentaje de } \\
\text { Anatasa }(\%)\end{array}$ & $\begin{array}{c}\text { Porcentaje de } \\
\text { Rutilo }(\%)\end{array}$ \\
\hline 10 & 52 & 65,70 & 34,30 \\
& 79 & 69,94 & 30,06 \\
20 & 52 & 75,74 & 24,26 \\
& 79 & 32,98 & 67,02 \\
30 & 52 & 26,80 & 73,20 \\
& 79 & 51,45 & 48,55 \\
\hline \hline
\end{tabular}

C. Medición del calor específico de láminas de Ti recubiertas con $\mathrm{S}-\mathrm{TiO}_{2}$ por $\mathrm{OEP}$.

Las muestras se sometieron a la técnica de relajación térmica para determinar su $C_{p}$. Se obtuvieron diferencias significativas de este parámetro, relacionadas con la concentración del electrolito, pero no con el ciclo útil de la tensión aplicada. En la Tabla V se observan los valores obtenidos.

a)

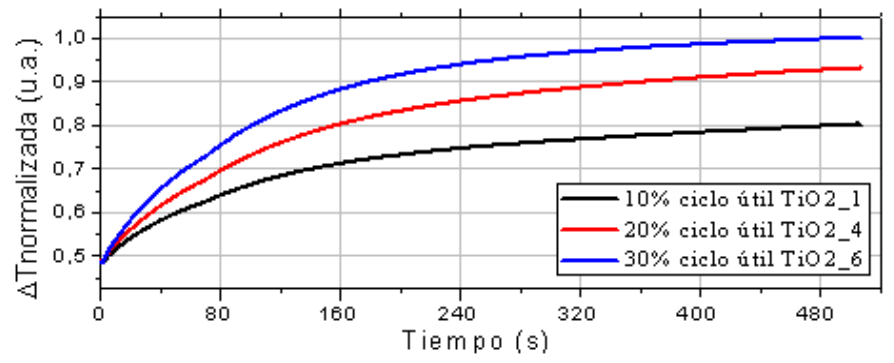

b)

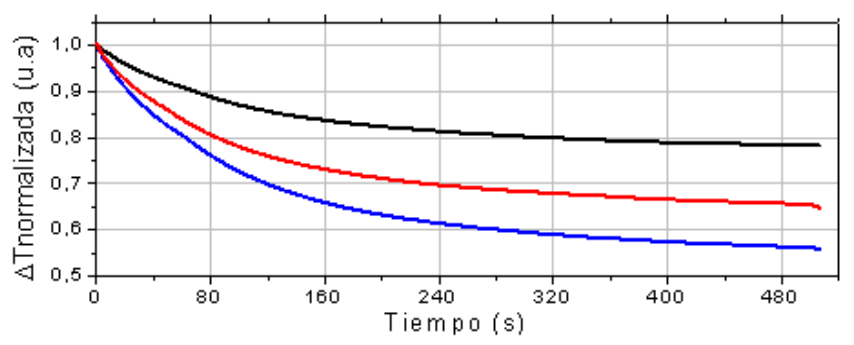

Fig. 4. Gradiente de temperatura de las muestras descritas en la Tabla I, en función del tiempo. Medida realizada en vacío con a) incidencia de luz láser y b) sin radiación. 
En la Fig. 4 se muestra la evolución del gradiente de temperatura de las muestras de Ti recubiertas con $\mathrm{S}-\mathrm{TiO}_{2}$ por OEP, descritas en la Tabla I, en función del tiempo, con incidencia de luz láser (calentamiento) y sin ella (enfriamiento).

TABLE V

$C_{P}$ DE LAS LÁMINAS RECUBIERTAS CON S-TiO 2 .

\begin{tabular}{ccc}
\hline \hline $\begin{array}{c}\text { Ciclo útil } \\
(\%)\end{array}$ & $\begin{array}{c}\text { Concentración } \\
(\mathrm{mM})\end{array}$ & $\begin{array}{c}\text { Calor específico } \\
\left(\mathrm{J} \cdot \mathrm{g}^{-1} \cdot \mathrm{K}^{-1}\right)\end{array}$ \\
\hline 10 & 52 & $0,39 \pm 0,01$ \\
& 79 & $0,42 \pm 0,02$ \\
20 & 52 & $0,38 \pm 0,02$ \\
& 79 & $0,43 \pm 0,02$ \\
30 & 79 & $0,44 \pm 0,02$ \\
& 52 & $0,38 \pm 0,01$ \\
\hline \hline
\end{tabular}

D. Aplicación de ANOVA de un solo factor a los valores $C_{p}$ de las muestras, correspondientes a variación de concentración del electrolito durante la OEP.

Se analizaron los valores de $C_{p}$ de las muestras recubiertas con $\mathrm{S}-\mathrm{TiO}_{2}$, en relación con la variación de la concentración de $\mathrm{CH}_{4} \mathrm{~N}_{2} \mathrm{~S}$. En la Tabla VI se muestran los valores promedio obtenidos, con su respectiva desviación estándar y coeficiente de variación. El coeficiente de variación se relaciona con la precisión del instrumento de medición y la homogeneidad de los datos. El valor promedio del $C_{p}$ de las muestras, crecidas con concentraciones de $52 \mathrm{mM}$ y $79 \mathrm{mM}$, fue de $0,386 \pm 0,008$ $\mathrm{J} \cdot \mathrm{g}^{-1} \cdot \mathrm{K}^{-1}$ y $0,428 \pm 0,008 \mathrm{~J} \cdot \mathrm{g}^{-1} \cdot \mathrm{K}^{-1}$, respectivamente.

TABLE VI

$C_{P}$ DE LAS LÁMINAS RECUBIERTAS CON $\mathrm{S}_{-} \mathrm{TIO}_{2}$, CONSIDERANDO LA VARIACIÓN DE LA CONCENTRACIÓN DE $\mathrm{CH}_{4} \mathrm{~N}_{2} \mathrm{~S}$ EN EL ELECTROLITO.

\begin{tabular}{ccccc}
\hline $\begin{array}{c}\text { Concentra } \\
\text { ción }\end{array}$ & Recuento & $\begin{array}{c}\text { Promedio } \\
\left(\mathrm{J} \mathrm{g}^{-1} \cdot \mathrm{K}^{-1}\right)\end{array}$ & $\begin{array}{c}\text { Desviación } \\
\text { Estándar } \\
\left(\mathrm{J} \cdot \mathrm{g}^{-1} \cdot \mathrm{K}^{-1}\right)\end{array}$ & $\begin{array}{c}\text { Coeficien } \\
\text { te de } \\
\text { Variación } \\
(\%)\end{array}$ \\
\hline 52 & 6 & 0,386 & 0,008 & 2,08 \\
79 & 6 & 0,428 & 0,008 & 2,02 \\
Total & 12 & 0,407 & 0,020 & 5,66 \\
\hline \hline
\end{tabular}

El ANOVA de un factor sirve para comparar las medias de los resultados del parámetro medido. Para determinar el potencial de discriminación con el $C_{p}$, se planteó una hipótesis nula $H_{o}$ y una hipótesis alternativa $H_{A}$ :

$$
H_{o}: C_{p_{52 m M}}=C_{p_{79 m M}}=C_{p}
$$

La hipótesis nula define que la media del $C_{p}$ de las muestras crecidas con concentración de $52 \mathrm{mM}$ y $79 \mathrm{mM}$ de $\mathrm{CH}_{4} \mathrm{~N}_{2} \mathrm{~S}$, son iguales; mientras que con la hipótesis alternativa $H_{A}$ de (11), el valor del parámetro $C_{p}$ es diferente en los dos casos.

$$
H_{A}: C_{p_{52 m M}} \neq C_{p_{79 m M}} \neq C_{p}
$$

TABLE VII

ANOVA APLICADO AL $C_{P}$ DE LAS LÁMINAS RECUBIERTAS CON $\mathrm{S}_{-} \mathrm{TIO}_{2}$, RELACIONADO CON LA VARIACIÓN DE LA CONCENTRACIÓN DE $\mathrm{CH}_{4} \mathrm{~N}_{4} \mathrm{~S}$ EN EL ELECTROLITO.

\begin{tabular}{lccccc}
\hline \hline Fuente & $\begin{array}{c}\text { Suma de } \\
\text { Cuadrados } \\
\left(\mathrm{J} \cdot \mathrm{g}^{-1} \cdot \mathrm{K}^{-1}\right)\end{array}$ & $\begin{array}{c}\text { Grados } \\
\text { de } \\
\text { libertad }\end{array}$ & $\begin{array}{c}\text { Cuadrado } \\
\text { Medio }\left(\mathrm{J} \cdot \mathrm{g}^{-}\right. \\
\left.1 \cdot \mathrm{K}^{-1}\right)\end{array}$ & $\begin{array}{c}\text { Razón } \\
-\mathrm{F}\end{array}$ & $\begin{array}{c}\text { Valor- } \\
\mathrm{P}\end{array}$ \\
\hline $\begin{array}{l}\text { Entre } \\
\text { grupos }\end{array}$ & 0,0052 & 1 & 0,0051 & 73,78 & 0,00 \\
$\begin{array}{l}\text { Intra } \\
\text { grupos }\end{array}$ & 0,0007 & 10 & $6,98 \times 10^{-5}$ & & \\
$\begin{array}{l}\text { Total } \\
\text { (Corr. })\end{array}$ & 0,0058 & 11 & & & \\
\hline \hline
\end{tabular}

En la Tabla VII se muestran los resultados del ANOVA; se observa que la variación total de los 12 datos analizados fue de $0,0058 \mathrm{~J} \cdot \mathrm{g}^{-1} \cdot \mathrm{K}^{-1}$. Al ponderar estos valores con los correspondientes grados de libertad, se obtuvieron los cuadrados medios que reflejan la magnitud real de cada fuente de variación. Se nota que la diferencia debida a la concentración utilizada de $\mathrm{CH}_{4} \mathrm{~N}_{2} \mathrm{~S}$ es de $0,0052 \mathrm{~J} \cdot \mathrm{g}^{-1} \cdot \mathrm{K}^{-1}$ y que el error es de $6,98 \times 10^{-5} \mathrm{~J} \cdot \mathrm{g}^{-1} \cdot \mathrm{K}^{-1}$; por lo tanto, el cuadrado medio de la concentración utilizada es aproximadamente 74 veces mayor que el cuadrado medio del error. Esto indica que las diferencias observadas entre las dos cantidades de concentraciones son significativas y que no se deben a pequeñas variaciones de las muestras. Como el valor $\mathrm{P}$ es menor del $5 \%$ se rechaza la hipótesis nula y se concluye que no hay igualdad entre las medias para cada cantidad de concentración utilizada de $\mathrm{CH}_{4} \mathrm{~N}_{2} \mathrm{~S}$. Para comprobar la presencia de diferencias significativas entre las medias del $C_{p}$ de las muestras, se probó la hipótesis utilizando el método de mínima diferencia significativa (LSD).

TABLE VII

VALORES RESULTANTES DE LA APLICACIÓN DE LA PRUEBA LSD DE ACUERDO CON LAS CONCENTRACIONES USADAS EN LOS CRECIMIENTOS.

\begin{tabular}{cccc}
\hline \hline Contraste & Sig & Diferencia & +/- Límites \\
\hline $52 \mathrm{mM}-79 \mathrm{mM}$ & 1 & 0,0414 & 0,0108 \\
\hline \hline
\end{tabular}

En la Tabla VII se observan los datos arrojados por la prueba LSD, de los que se determina que las medias son significativamente diferentes con un nivel del $95 \%$ de confianza.

E. Aplicación de ANOVA de un solo factor a los valores $C_{p}$ de las muestras, correspondientes a variación del ciclo útil.

En la Tabla VIII se muestran los valores promedio obtenidos con su respectiva desviación estándar y coeficiente de variación. El valor promedio del $C_{p}$ de las muestras, 
relacionas con variación del ciclo útil durante la OEP, fue de $0,40 \pm 0,02 \mathrm{~J} \cdot \mathrm{g}^{-1} \cdot \mathrm{K}^{-1}, 0,41 \pm 0,03 \mathrm{~J} \cdot \mathrm{g}^{-1} \cdot \mathrm{K}^{-1}$, y $0,41 \pm 0,03 \mathrm{~J} \cdot \mathrm{g}^{-1} \cdot \mathrm{K}^{-1}$.

TABLE VIII

$C_{P}$ DE LAS LÁMINAS RECUBIERTAS CON S-TiO 2 , CONSIDERANDO LA VARIACIÓN DEL CICLO ÚTIL DURANTE LA OEP.

\begin{tabular}{ccccc}
\hline \hline $\begin{array}{c}\text { Ciclo } \\
\text { útil } \\
(\%)\end{array}$ & Recuento & $\begin{array}{c}\text { Promedio } \\
\left(\mathrm{J} \cdot \mathrm{g}^{-1} \cdot \mathrm{K}^{-1}\right)\end{array}$ & $\begin{array}{c}\text { Desviación } \\
\text { Estándar } \\
\left(\mathrm{J} \cdot \mathrm{g}^{-1} \cdot \mathrm{K}^{-1}\right)\end{array}$ & $\begin{array}{c}\text { Coeficiente } \\
\text { de Variación } \\
(\%)\end{array}$ \\
\hline 10 & 4 & 0,40 & 0,02 & 4,31 \\
20 & 4 & 0,41 & 0,03 & 7,28 \\
30 & 4 & 0,41 & 0,03 & 6,49 \\
Total & 12 & 0,4076 & 0,02 & 5,66 \\
\hline \hline
\end{tabular}

Como en el caso anterior, se planteó una hipótesis nula $H_{o}$ y una hipótesis alternativa $H_{A}$ :

$$
H_{o}: C_{p_{10 \%}}=C_{p_{20 \%}}=C_{p_{30} \%}=C_{p}
$$

La hipótesis nula descrita en (12) define que las medias del $C_{p}$ de las muestras crecidas con 10, 20 y $30 \%$ de ciclo útil son iguales; mientras que la hipótesis alternativa $H_{A}$ de (13) propone que el valor del $C_{p}$ es diferente en los tres casos:

$$
H_{A}: C_{p_{10 \%}} \neq C_{p_{20 \%}} \neq C_{p_{30} \%} \neq C_{p}
$$

TABLE IX

ANOVA APLICADO AL C $C_{P}$ DE LAS LÁMINAS RECUBIERTAS CON S-TiO 2 , RELACIONADO CON LA VARIACIÓN DEL CICLO ÚTIL.

\begin{tabular}{lccccc}
\hline \hline Fuente & $\begin{array}{c}\text { Suma de } \\
\text { Cuadrados } \\
\left(\mathrm{J} \cdot \mathrm{g}^{-1} \cdot \mathrm{K}^{-1}\right)\end{array}$ & Gl & $\begin{array}{c}\text { Cuadrado } \\
\text { Medio }\left(\mathrm{J} \cdot \mathrm{g}^{-}\right. \\
\left.1 \cdot \mathrm{K}^{-1}\right)\end{array}$ & $\begin{array}{c}\text { Razón- } \\
\mathrm{F}\end{array}$ & $\begin{array}{c}\text { Valor- } \\
\mathrm{P}\end{array}$ \\
\hline $\begin{array}{l}\text { Entre } \\
\text { grupos }\end{array}$ & 0,00017 & 2 & $8,538 \mathrm{X} 10^{-5}$ & 0,14 & 0,8752 \\
$\begin{array}{l}\text { Intra } \\
\text { grupos }\end{array}$ & 0,00567 & 9 & 0,00063 & & \\
$\begin{array}{l}\text { Total } \\
\text { (Corr.) }\end{array}$ & 0,00585 & 11 & & & \\
\hline \hline
\end{tabular}

En la tabla IX se muestran los resultados del ANOVA; se observa que la variación total de los datos analizados fue de $0,00585 \mathrm{~J} \cdot \mathrm{g}^{-1} \cdot \mathrm{K}^{-1}$. Se nota que el cuadrado medio es aproximadamente 0,14 veces mayor que el cuadrado medio del error; esto indica que las diferencias observadas entre los tres ciclos útiles no son significativas y se aprueba la hipótesis nula con un nivel del $5 \%$ de significación, debido a que el valor P es mayor del $5 \%$; por lo que hay igualdad entre las medias para cada variación del ciclo útil.

\section{CONCLUSIONES}

Mediante la técnica de relajación térmica se halló el $C_{p}$ de láminas de Ti recubiertas con $\mathrm{S}-\mathrm{TiO}_{2}$ por OEP. El ANOVA de un solo factor, aplicado a los datos, cuya variable respuesta fue el valor de este parámetro, llevó a establecer que el $C_{p}$ de las muestras crecidas con el uso de $\mathrm{CH}_{4} \mathrm{~N}_{2} \mathrm{~S}$ a $52 \mathrm{mM}$ y a 79 $\mathrm{mM}$ es aproximadamente $0,386 \mathrm{~J} \cdot \mathrm{g}^{-1} \cdot \mathrm{K}^{-1}$ y $0,428 \mathrm{~J} \cdot \mathrm{g}^{-1} \cdot \mathrm{K}^{-1}$, respectivamente.

El $C_{p}$ de las muestras no presentó modificaciones relacionadas con la variación del ciclo útil durante el crecimiento por OEP, posiblemente debido a la influencia determinante que tuvo este parámetro en el porcentaje de fase Anatasa y Rutilo, que conlleva a cambios en la de densidad de la muestra. Por otro lado, se encontró una dependencia de este valor con la cantidad de impureza agregada al recubrimiento; lo que sugiere que la concentración de $\mathrm{CH}_{4} \mathrm{~N}_{2} \mathrm{~S}$ no afecta significativamente el tamaño de grano, la porosidad, ni la fase de la muestra.

\section{AGRADECIMIENTOS}

Los autores agradecen a la Universidad del Quindío por la financiación de este trabajo a través del proyecto 924 "Evaluación de características mecánicas y térmicas de elementos estructurales elaborados con guadua Angustifolia Kunth y con un adhesivo sintetizado con aceite de higuerilla".

\section{REFERENCIAS}

[1] E. Marín, O. Delgado-Vasallo and H. Valiente, "A temperature relaxation method for the measurement of the specific heat of solids at room temperature in student laboratories," American Association of Physics Teachers, vol. 71, no. 10, pp. 1032-1036, Octubre 2003. DOI: 10.1119/1.1586261.

[2] N. A. Lara Bernal, "Método de calentamiento transitorio para la caracterización térmica de sólidos". Ph.D. tesis, Instituto Politécnico Nacional, México, 2013.

[3] S. García, E. Marín, O. Delgado-Vasallo, J. Portelles, G. Peña Rodríguez, A. Calderón, E. Martinez y J. M. Siqueiros, "Thermal properties of $\mathrm{Sr}_{0,3} \mathrm{Ba}_{0,7} \mathrm{Ti}_{1-y} \mathrm{Zr}_{\mathrm{y}} \mathrm{O}_{3}$ ferroelectric ceramics: Dependence on sample's porosity," Journal Physics IV France, vol. 125, pp. 309-311, Diciembre 2005. DOI: 10.1051/jp4:2005125074.

[4] J. Alexandre, F. Saboya, B. C. Marques, M. L. P. Ribeiro, C. Salles, M. G. da Silva, M. S. Sthel, L. T. Auler and H. Vargas, "Photoacoustic thermal characterization of kaolinite clays, The Analyst," vol. 124, pp.1209-1214, Agosto 1999. DOI: 10.1039/a902601k.

[5] A. Lara-Bernal, E. Marín, and A. Calderón, "Técnica de relajación térmica con excitación variable: Caso de la "rampa' de intensidad," Superficies y vacío, vol. 20, no. 3, pp. 17-20, Junio 2007, ISSN 1665-3521.

[6] S. B. Luo, W. L. Wang, J. Chang, Z. C. Xia, and B. Wei, "Specific heat capacity of liquid and solid $\mathrm{Ni}_{83.5} \mathrm{Ti}_{16.5}$ eutectic alloy, Chemical," Physics Letters., vol. 679, pp. 172-175, Julio 2017. DOI: 10.1016/j.cplett.2017.04.053.

[7] H. Valiente, O. Delgado-Vasallo, R. Abdelarrague, A. Calderón, and E. Marin, "Specific heat measurements by a thermal relaxation method: Influence of convection and conduction, International," Journal Thermophysics., vol. 27, no. 6, pp. 1859-1872, Enero 2006. DOI: 10.1007/s10765-006-0127-1.

[8] R. Quick, J.E.K. Schawe, P.J. Uggowitzer and S. Pogatscher, "Measurement of specific heat capacity via fast scanning calorimetry-Accuracy and loss corrections," Thermochimica Acta, vol. 677, pp. 12-20, Julio 2019. DOI: 10.1016/j.tca.2019.03.021.

[9] S.S. Gonçalves, "Novel alginate-chitosan aerogel fibers for potential wound healing applications", conference: 8th international conference and exhibition on pharmaceutics \& novel 
drug delivery systems, Madrid, España, Marzo 2016. DOI: 10.4172/2153-2435.C1.027-032.

[10] A. Rosencwaig and A. Gersho, "Theory of the photoacoustic effect with solids," Journal of Applied Physics. vol. 47, no. 64, p. 52, 1976.

[11] G. Seidel and P. H. Keesom, "Specific heat of gallium and zinc in the normal and superconducting states," Physical Review, vol. 12, no. 4, pp. 1083-1088, Noviembre 1958. DOI: 10.1103/PhysRev.112.1083.

[12] M. M. Yakunkin, "Mechanism of High-Temperature Heat Capacity of Tungsten : Study of Relaxation Processes," Physics of the Solid State, vol. 46, no. 2, pp. 219-225, Febrero 2004. DOI: 10.1134/1.1649413.

[13] J. M. González, "Producción de películas de nitruro-titanioaluminio- vanadio (tialv)n variando la temperatura del sustrato por la técnica papvd", M.S thesis. Universidad nacional de Colombia, sede Manizales, Colombia, 2007.

[14] F. Incropera and D. Dewitt. Fundamentos de transferencia de calor. ED-4, Prentice Hall, México, 1999. ISBN: 970-17-0170-4.

[15] Y. Wang, J. Li, P. Peng, T. Lu, and L. Wang, "Preparation of S$\mathrm{TiO}_{2}$ photocatalyst and photodegradation of $\mathrm{L}$-acid under visible light," Applied Surface Science., vol. 254, no. 16, pp. 5276-5280, Junio 2008. DOI: 10.1016/j.apsusc.2008.02.050.

[16] R. A. Spurr and H. Myers, "Quantitative analysis of anatase-rutile mixtures with an x-ray diffractometer," Analytical Chemistry., vol. 29, no. 5, pp. 760-762, Mayo 1957. DOI: 10.1021/ac60125a006.

[17] R. Bacsa, J. Kiwi, T. Ohno, P. Albers, and V. Nadtochenko, "Preparation, testing and characterization of doped $\mathrm{TiO}_{2}$ active in the peroxidation of biomolecules under visible light," Journal of Physical Chemistry B, vol. 109, no. 12, pp. 5994-6003, Marzo 2005. DOI: $10.1021 / \mathrm{jp} 044979 \mathrm{c}$.

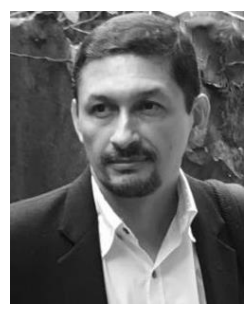

Fernando Gordillo-Delgado es licenciado en matemáticas y física, egresado de la Universidad del Tolima (Colombia). Hizo su especialización en física en este mismo centro educativo y obtuvo su título de maestría en ciencias con especialización en física en el Centro de Investigación y de Estudios Avanzados del IPN (México). Cursó sus estudios de doctorado en el Centro de Investigación en Ciencia Aplicada y Tecnología Avanzada del IPN (México). Actualmente es docente de la Universidad del Quindío (Colombia). Su trabajo de investigación ha estado orientado hacia la aplicación de nuevos materiales y técnicas de caracterización fototérmicas al desarrollo de la producción agrícola más limpia.

ORCID: https://orcid.org/0000-0002-2940-1129

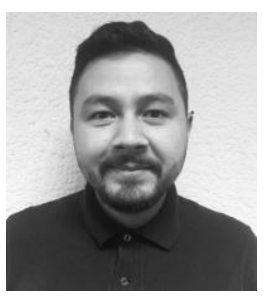

Diego Fernando Valencia Grisales, nació en Cali, Valle del Cauca, Colombia. Obtuvo su título de Ingeniero Electrónico de la Universidad del Quindío (UQ) en abril del 2019. Desarrolló su trabajo de grado en el GICADE de la UQ, en el cual diseñó e implementó un sistema para medir calor específico en muestras sólidas mediante radiación de luz láser y termometría infrarroja. Sus áreas de interés están ligadas a la aplicación del efecto fotoacústico y a la caracterización térmica de los materiales. Actualmente cursa sus estudios de maestría en el Instituto Nacional de Astrofísica Óptica y Electrónica (INAOE) en Puebla, México.
ORCID: https://orcid.org/0000-0002-5555-9210

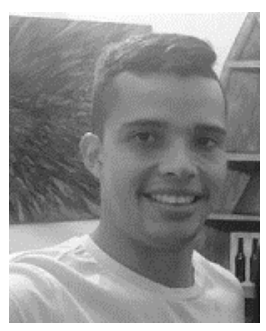

John Alexander García Giraldo, nacido en Armenia Colombia es ingeniero electrónico egresado de la Universidad del Quindío (2014). Ha sido dos veces Joven Investigador de Colciencias (2015 y 2018) y joven investigador en la misma Universidad de graduación (2017), docente catedrático en el programa de Ingeniería electrónica (2016) y docente en el técnico de instalaciones eléctricas el Instituto técnico Comfenalco Quindío (2017). Su trabajo de grado se enfocó en el diseño e instrumentación de un proceso térmico para la obtención de carbón activado a partir de la guadua. Sus trabajos como joven investigador se enfocan en la medición de parámetros térmicos de productos biológicos y químicos, programación de sistemas embebidos y diseño de circuitos eléctricos y electrónicos CAD y PCB. Integrante del GICADE, de la Universidad del Quindío.

ORCID: https://orcid.org/0000-0002-2628-7850 